Journal of Computer Science 6 (9): 1042-1047, 2010

ISSN 1549-3636

(C) 2010 Science Publications

\title{
Iris Recognition Without Iris Normalization
}

\author{
${ }^{1}$ Lenina Birgale and ${ }^{2} \mathrm{M}$. Kokare \\ ${ }^{1}$ Department of Electronics and Telecommunication Engineering, \\ Institute of Chartered Financial Analysts of India University, \\ Institute of Science and Technology, 4-102, Jeedimetla, NH 7, \\ Hyderabad, Andhra Pradesh State, 500055, India \\ ${ }^{2}$ Department of Electronics and Telecommunication Engineering, \\ Shri Guru Gobind Singhji Institute of Engineering and Technology, \\ Vishnupuri, Nanded, Maharashtra State, 431606, India
}

\begin{abstract}
Problem statement: In any real time biometric system processing speed and recognition time are crucial parameters. Reducing processing time involves many parameters like normalization, FAR, FRR, management of eyelid and eyelash occlusions, size of signature etc. Normalization consumes substantial amount of time of the system. This study contributes for improved iris recognition system with reduced processing time, False Acceptance Rate (FAR) and False Rejection Rate (FRR). Approach: To improve system performance and reliability of a biometric system. It avoided the iris normalization process used traditionally in iris recognition systems. The technique proposed here used different masks to filter out iris image from an eye. Comparative study of different masks was done and optimized mask is proposed. The experiment was carried on CASIA database consisting of 756 iris images of 108 persons. Each person contributes seven images of eye $(108 \times 7=$ 756) images in the database. Results: In the proposed method: (1) Normalization step is avoided; (2) Computational time is reduced by $0.3342 \mathrm{sec}$; (3) Iris signature size is reduced; (4) Improved performance parameters. (With reduced feature size, proposed method achieves $99.4866 \%$ accuracy, 0.0069\% FAR, 1.0198\% FRR and significant increase in speed of the system). Conclusion: Iris signature proposed was comparatively small just of $1 \times 24$ size. Though Daugman's method gives best accuracy of $99.90 \%$ but the iris signature length used by that algorithm is comparatively very high that is $1 \times 2048$ phase vector. Also Daugman has used phase information in signature formation. Our method gives a accuracy of $99.474 \%$ with a signature of comparatively very small length. This has definitely contributed to improve the speed.
\end{abstract}

Key words: Normalization, biometrics, features, database, mask, wavelets, FAR, FRR, Euclidian distance

\section{INTRODUCTION}

Security system is the need of the day. Fastest recognition system is the demand of current situation. Facial features, voice patterns, hand geometry, retinal patters, vein patterns, signature dynamics, voice verification, facial thermography, DNA matching nailbed identification, gait recognition, earshape recognition and finger prints have all been explored as biometric identifiers with varying levels of success. However iris being, unique and stable for a life period is the most reliable biometric. Iris as recognition biometric for identification formed the active research area since 1992. The uniqueness of iris patterns was identified since then. This uniqueness property of iris can be quoted in the words of Daugman (1993) as, 'An advantage of the iris shares with fingerprints is the chaotic morphogenesis of its minutiae'. Iris can be identified as the colored portion of the eye lying between pupil and sclera. A front-on view of the human eye is shown in Fig. 1a. A very important characteristic of an iris is that it is a naturally protected organ and is stable without any variations along with the age of an individual. The main motivation of this study is the processing time required to process the query with traditional normalization method, the problems faced

Corresponding Author: Lenina Birgale, Department of Electronics and Telecommunication Engineering,

Institute of Chartered Financial Analysts of India University, Institute of Science and Technology,

4-102, Jeedimetla, NH 7, Hyderabad, Andhra Pradesh State, 500055, India 
by eyelid-eyelash occlusions and multi resolution provided by wavelets as verified by our previous study (Birgale et al., 2006; Birgale and Kokare, 2009). It helps to compare speed of the recognition system with and without iris normalization.

Various research groups such as Ma et al. (2002); Tisse et al. (2002); Zhu et al. (2000) and Wildes et al. (1996) has proposed a recognition system based on iris normalization. Rydgren et al. (2004) has proposed a system based on iris normalization. The iris normalization step has become so popular in the research groups that it is being called as Daugman's rubber sheet model after the name of renowned researcher Daugman. Iris segmentation is shown in Fig. $1 \mathrm{~b}$ and its normalization is shown in Fig. 1c. Our study shows that if we avoid iris normalization we are able to save comparable amount of processing and recognition time.

The main contributions of this study are summarized as follows:

- Use of a specially designed mask which avoids eyelid and eyelash occlusions.

- Reduction in signature size.

- Improvement in processing speed.

We use standard wavelets to extract texture information required in iris signature formation. Here, we have used three level decomposition of the iris signature. It gives better results. Obtained results are compared with the previous method (Chu and Chen, 2005; Daugman, 1993; 2001; Cui et al., 2004; Birgale and Kokare, 2009; Ma et al., 2002; Maheswari et al., 2008; De Martin-Roche et al., 2001). The mask optimization avoids eyelid and eyelash occlusions.

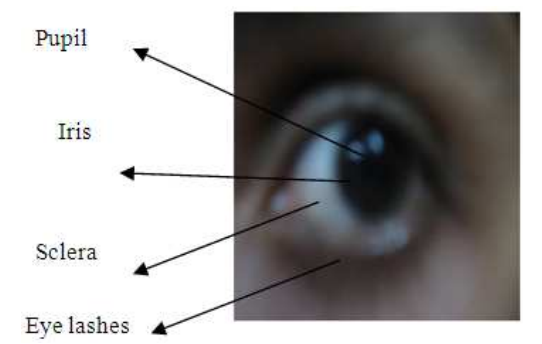

(a)

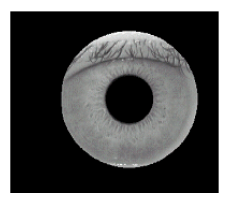

(b)
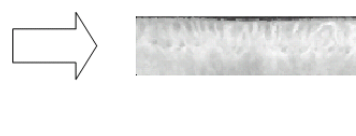

(c)

Fig. 1: (a) A front-on view of the human eye; (b) Segmented iris; (c) Normalized iris
This study can be outlined as follows. A brief overview of eyelid and eyelash occlusion is presented followed by a brief account of Discrete Wavelet transforms. Then we lime light the proposed method followed by experimental results. An elaborate discussion of the proposed method is followed by conclusion.

Proposed IRIS masks: Eyelid and eyelash occlusions form the major noise component in an iris recognition system. It needs to be managed in a careful way to minimize FAR and FRR. Basically eyelid and eyelash occlusions cause major disturbances in partially opened eyes from upper and lower side of an iris as shown in Fig. 2a. Figure $2 b$ and $c$ shows that eyelid and eyelash occlusions can be avoided by use of proper mask for segmentation. This avoids the unnecessary processing. We used Discrete Wavelet Transform (DWT) for feature extraction.

The discrete wavelet transform: The Short Time Fourier Transform (STFT) represents a sort of compromise between the time-and frequency-based views of a signal. It provides some information about both when and at what frequencies a signal event occurs. However, one can only obtain this information with limited precision and that precision is determined by the size of the window. But many signals require a more flexible approach where we can vary the window size to determine more accurately either time or frequency. Wavelet analysis is such an approach in which window with variable-sized regions is used. Wavelet analysis allows the use of long time intervals where we want more precise low-frequency information and shorter regions where we want high-frequency information. However it is a time-frequency region, but rather a time scale.

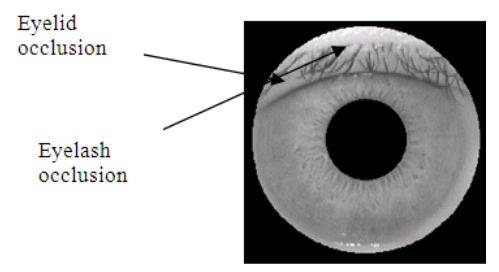

Fig. 2a: Segmented Iris with eyelid and eyelash occlusions

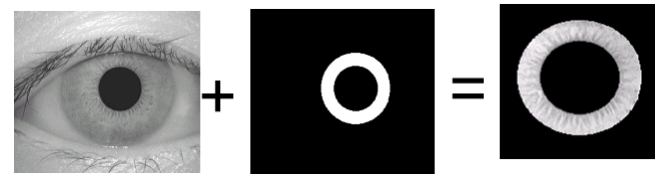

Fig. 2b: Process of iris segmentation with circular mask 
Computing wavelet coefficients at every possible scale is a fair amount of study and it generates an awful lot of data. That is why we choose only a subset of scales and positions at which to make our calculations. It turns out, rather remarkably, that if we choose scales and positions based on powers of two so-called dyadic scales and positions then our analysis will be much more efficient and just as accurate. We obtain such an analysis from the Discrete Wavelet Transform (DWT) given by (1):

$$
\text { DWT }=\sum_{\mathrm{k}=1}^{\infty} \sum_{1=-\infty}^{+\infty} \mathrm{q}(\mathrm{k}, 1) \psi\left(2^{-\mathrm{k}} \mathrm{t}-1\right)
$$

An efficient way to implement this scheme using filters was developed in 1988. This algorithm is in fact a classical scheme known in the signal processing community as a two-channel sub band coder. This very practical filtering algorithm yields a fast wavelet transform a box into which a signal passes and out of which wavelet coefficients quickly emerge. Let's examine this in more depth.

Let:

$$
\begin{aligned}
& \phi(x)=\sum_{n} h_{\varphi}(n) \sqrt{2} \phi(2 x-n) \\
& \psi(x)=\sum_{n} h_{\psi}(n) \sqrt{2} \psi(2 x-n)
\end{aligned}
$$

Both $\phi(x)$ and $\psi(x)$ can be expressed as linear combinations of double-resolution copies of themselves. Here $h_{\phi}$ in (2) and $h_{\psi}$ in (3) the expansion coefficients are called scaling and wavelet vectors, respectively. They are the filter coefficients of Fast Wavelet Transform (FWT):

- $\mathrm{W}_{\phi}(\mathrm{j}, \mathrm{m}, \mathrm{n})$ Approximate coefficients

- $\mathrm{W}_{\psi}^{\mathrm{H}}(\mathrm{j}, \mathrm{m}, \mathrm{n})$ Horizontal coefficients

- $\mathrm{W}_{\psi}^{\mathrm{v}}(\mathrm{j}, \mathrm{m}, \mathrm{n})$ Vertical coefficients

- $\quad \mathrm{W}_{\psi}^{\mathrm{D}}(\mathrm{j}, \mathrm{m}, \mathrm{n})$ Diagonal coefficients

Here $W_{\phi}(j, m, n)$ is the original image whose DWT is to be computed.
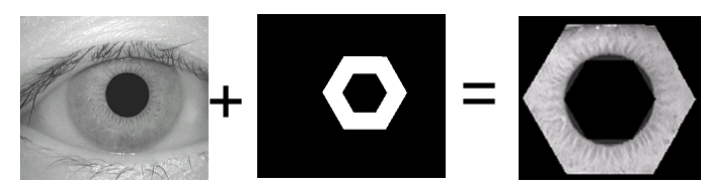

Fig. 2c: Process of iris segmentation with hexagonal mask

\section{MATERIALS AND METHODS}

Proposed method: The proposed method gives better performance in terms of accuracy, FAR, FRR and speed. It reduces the false acceptance rate and false rejection rate and improves the system efficiency with minimum process and recognition time. The feature vector size used by us is very small as compared to that used by Daugman (2001) which also uses iris normalization. Our method skips this normalization. It is discussed below.

Image database: The database used in the experimentation consists of 756 different iris images from CASIA iris image database. CASIA iris database is the only public domain database available. Size of each database iris image is $280 \times 320$. In this database there are 108 different subjects. Each subject has contributed to seven different images of same eye. Thus there are a total of $756(108 \times 7)$ images in the database. Among the seven images three are taken in first session and other four are taken in second session.

Feature database creation: Each eye image is filtered out by using the masks designed. Three level wavelet decomposition is obtained for every filtered image as shown in Fig. 3. We have used both Daubchies and Haar wavelet coefficients and shown the comparative results. To compare results obtained by normalization we have designed different masks. These masks are used to avoid normalization step.

To calculate the signature of iris we have used first level energy (4) and computed standard deviation (5) of each sub-band. Thus the signature vector size of database is of $756 \times 24$ and for query image it is of size $1 \times 24$. First level energy is computed by (4):

$$
\text { Energy }=\sum_{0}^{M-1} \sum_{0}^{N-1}|X(m, n)|
$$

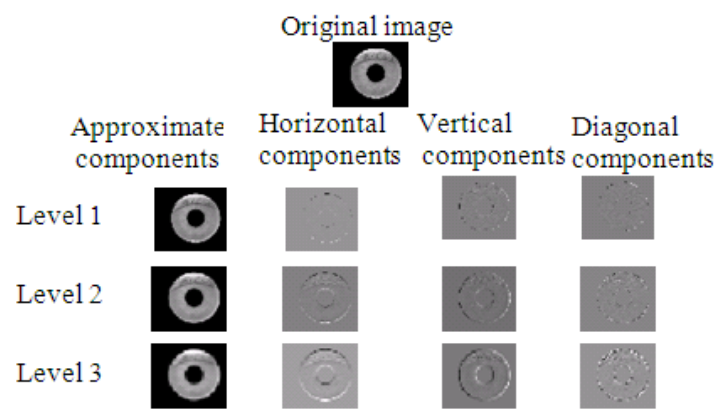

Fig. 3: Three level decomposition of segmented iris 
where, $\mathrm{X}(\mathrm{m}, \mathrm{n})$ is a discrete function whose energy is to be computed.

Similarly standard deviation is given by (5):

$$
\sigma_{k}=\frac{1}{M X N} \sum_{i=1}^{N} \sum_{j=1}^{N} E\left[W_{k}(i, j)-\mu_{k}\right]
$$

Where:

$\mathrm{W}(\mathrm{i}, \mathrm{j})=$ The $\mathrm{k}^{\text {th }}$ wavelet decomposed sub-band

$\mathrm{M} \times \mathrm{N}=$ The wavelet decomposed sub-band

$\mu_{\mathrm{k}} \quad=$ The mean value of kth decomposed sub-band

Matching: A query signature is one of 756 signatures from image database signatures. Euclidean distance metric given by (6) is used to compute the similarity or match value for given pair of signatures. Zero distance implies a perfect match and signature tends towards mismatch as the distance increases:

$$
\mathrm{D}_{(\mathrm{x}, \mathrm{y})}^{\text {Eucli }}=\sqrt{\sum_{\mathrm{i}=0}^{\mathrm{N}}\left(\mathrm{x}_{\mathrm{i}}-\mathrm{y}_{\mathrm{i}}\right)^{2}}
$$

Performance measures: The threshold value used is the average distance of top seven signatures as they belong to different eye images of same person's eye. Three images are taken in first session and four are taken in second session. The threshold thus set is used to compute speed of recognition, False Acceptance Rate (FAR) and False Rejection Rate (FRR). Here Speed of recognition, FAR and FRR are the performance measures used.

FAR is computed by (7) and FRR is computed by (8):

$\mathrm{FAR}=\frac{\mathrm{NFA}}{\mathrm{NIVA}}$

Where:

NFA $=$ Number of False Acceptances

NIVA $=$ Number of Imposter Verification Attempts

FAR $=$ The measure of likelihood that the biometric security system would incorrectly accept that an attempt by an unauthorized user

$\mathrm{FRR}=\frac{\mathrm{NFR}}{\mathrm{NEVA}}$

Where:

NFR $=$ Number of False Rejections

NEVA $=$ Number of Enrollee Verification Attempts

FRR = The measure of likelihood that the biometric security system would incorrectly reject that an attempt by an unauthorized user

\section{RESULTS}

Experimental results: Results obtained by the proposed method are much interesting and reliable. They are listed below in Table 1 and 2. Table 1 gives speed comparison with normalization and without normalization. From this we can see that we are able to achieve a time economy of $0.3342 \mathrm{sec}$ with the proposed method. Table 1 gives comparison of different algorithms and proposed method. It is evident from this Table that the results obtained by proposed method are comparatively much better than the existing methods though we have used signature of small size. Average efficiency obtained is $99.48 \%$. Thus it is evident from this that the results are comparatively outperforming over other methods and our previous method. The database used is digitized grayscale eye images from Chinese Academy of Sciences-Institute of Automation (2003) (CASIA).

Table 1: Time required to process and recognizes an iris with normalization and without normalization

\begin{tabular}{ll}
\hline Method used & Query process and recognition time (sec) \\
\hline With rubber sheet & 1.1740 \\
Without rubber sheet & 0.8398 \\
Time economy achieved & 0.3342 \\
\hline
\end{tabular}

Table 2: Comparison of results obtained with existing methods

\begin{tabular}{llll}
\hline Method used & FAR $(\%)$ & FRR (\%) & Average efficiency (\%) \\
\hline Our previous method (with normalization and daubchies wavelet) & 0.0071 & 1.041 & 98.950 \\
Our previous method (with normalization and haar wavelet) & 0.0069 & 1.021 & 98.980 \\
The proposed method (Without normalization and daubchies) & 0.0069 & 1.021 & 99.485 \\
The proposed method (Without normalization and haar wavelet) & 0.0069 & 1.020 & 99.486 \\
Optimized_circular_mask (Without normalization and daubchies) & 0.0072 & 1.060 & 99.469 \\
Optimized_circular_mask (Without normalization and haar) & 0.0072 & 1.050 & 99.471 \\
Optimized hex mask (Without normalization and daubchies) & 0.0072 & 1.050 & 99.471 \\
Optimized hex mask without normalization and (haar) & 0.0071 & 1.050 & 99.474 \\
\hline
\end{tabular}




\section{DISCUSSION}

Normalization of iris is significantly time consuming process. Even at the time of signature database creation, the system consumes lot of time for this process. The proposed reduction in processing time by $0.3342 \mathrm{sec}$ in a real time system contributes for improvement in system performance. This is achieved by skipping the classical normalization step. Mask optimization addresses the problem of eyelid and eyelash occlusions in conformation with efficiency. Comparison of different wavelet coefficients like haar and daubchies helps to select appropriate filters for a particular application.

\section{CONCLUSION}

From the listed Table 1 and 2 it can be concluded that the proposed method gives improvement in speed as well as average accuracy. Iris signature is comparatively small $1 \times 24$. Though Daugman's method gives best accuracy of $99.90 \%$ but the iris signature length used by that algorithm is comparatively very high that is $1 \times 2048$ phase vector. Also Daugman has used phase information in signature formation. Our method gives a accuracy of $99.474 \%$ with a signature of comparatively very small length. This has definitely contributed to improve the speed. However the proposed method can be further improved to obtain accuracy of $100 \%$.

\section{ACKNOWLEDGEMENT}

We are really grateful to Iris Recognition Research Group, Center for Biometrics and Security Research, National Laboratory of Pattern Recognition, Institute of Automation, Chinese Academy of Sciences for providing the database for research. We are also grateful to ICFAI Tech, Hyderabad, for the cordial atmosphere provided at the campus despite the busy academic year. Lastly but not least we are grateful to all the anonymous reviewers of this research.

\section{REFERENCES}

Birgale, L.V. and M. Kokare, 2009. Iris recognition using discrete wavelet transform. Proceedings of the IEEE International Conference, Mar. 7-9, IEEE Xplore Press, Bangkok, pp: 147-151. DOI: 10.1109/ICDIP.2009.30
Birgale, L., M. Kokare and D. Doye, 2006. Color and texture features for content based image retrieval. Proceedings of the 3rd IEEE International Conference Computer Graphics, Imaging and Visualization, July 26-28, IEEE Computer Society, Sydney, Australia, pp: 146-149. DOI: 10.1109/CGIV.2006.30

Chinese Academy of Sciences-Institute of Automation, 2003. Database of 756 greyscale eye images. Chinese Academy of Sciences-Institute of Automation.

Chu, C.T. and C.H. Chen, 2005. High performance iris recognition based on LDA and LPCC. Proceedings of the IEEE Conference on Tools with Artificial Intelligence, Nov. 16-16, IEEE Xplore Press, Hong Kong, pp: 417-421. DOI: 10.1109/ICTAI.2005.71

Cui, J., Y. Wang, J.Z. Huang, T. Tan and Z. Sun, 2004. An iris image synthesis method based on PCA and superresolution. Proceedings of the 17th International Conference on Pattern Recognition, Aug. 23-26, IEEE Xplore Press, USA., pp: 471-474. DOI: 10.1109/ICPR.2004.1333804

Daugman, J.G., 1993. High confidence visual recognition of persons by a test of analysis of statistical independence. IEEE Trans. Patt. Anal. Mach. Intel., 15: 1148-1161. DOI: $10.1109 / 34.244676$

Daugman, J., 2001. Statistical richness of visual phase information: Update on recognizing persons by iris patterns. Int. J. Comput. Vis., 45: 25-34. DOI: DOI: $10.1023 / \mathrm{A}: 1012365806338$

De Martin-Roche, D., C. Sanchez-Avila and R. SanchezReillo, 2001. Iris recognition for biometric identification using dyadic wavelet transform zerocrossing. Proceeding of the IEEE 35th Carnahan International Conference on Security Technology, Oct. 16-19, IEEE Xplore Press, London, UK., pp: 272-277. DOI: 10.1109/.2001.962844

Ma, L., Y. Wang and T. Tan, 2002. Iris recognition using circular symmetric filters. Proc. Int. Conf. Patt. Recog., 2: 414-417. DOI: 10.1109/ICPR.2002.1048327

Maheswari, S.U., P. Anbalagan and T. Priya, 2008. Efficient iris recognition through improvement in iris segmentation algorithm. ICGST-GVIP J., 8: 29-35.

http://www.icgst.com/gvip/volume8/Issue2/P11508 20002.pdf

Rydgren, E., E.A. Thomas, F. Amiel, F. Rossant and A. Amara, 2004. Iris features extraction using wavelet packets. Proceeding of the IEEE an International Conference on Image Processing, Oct. 24-27, IEEE Xplore Press, USA., pp: 861-864. DOI: 10.1109/ICIP.2004.1419435 
Tisse, C.L., L. Martin, L. Torres and M. Robert, 2002. Person identification technique using human iris recognition. Proceedings of the 15th International Conference on Vision Interface, May 27-29, IEEE Xplore Press, Calgary, Canada, pp: 294-300.

Wildes, R.P., J.C. Asmultr, G.L. Green, S.C. Hsu and R.J. Kolczynski et al., 1996. A machine-vision system for iris recognition. Mach. Vis. Appli., 9: 1-8. DOI: $10.1007 / \mathrm{BF} 01246633$
Zhu, Y., T. Tan and Y. Wang, 2000. Biometric personal identification based on iris patterns. Proceeding of the 15th International Conference on Pattern Recognition, Sept. 3-7, IEEE Xplore Press, Barcelona, Spain, pp: 801-804. DOI: 10.1109/ICPR.2000.906197 